\title{
An Ultra High Speed Digital 4-2 Compressor in 65-nm CMOS
}

\author{
Peiman Aliparast, Ziaadin D. Koozehkanani, and Farhad Nazari
}

\begin{abstract}
The presented work deals an ultra high-speed CMOS 4-2 compressor which is an essential part in fast digital arithmetic integrated circuits. Current-mode techniques have been used to improve the overall performance of the compressor. New fully differential proposed circuit improves delay to less than $37 \%$ also reduces occupied area in comparison to other high-speed conventional compressor circuits. To evaluate the performance of the proposed circuit, conventional gate level structure has been chosen and all of the circuits have been simulated in $65-\mathrm{nm}$ IBM CMOS process with $1.2 \mathrm{~V}$ power supply voltage.
\end{abstract}

Index Terms-Digital logic, 4-2 compressor, CMOS, high speed, current-mode.

\section{INTRODUCTION}

With ever-increasing possibilities that VLSI systems provide to realize high-speed digital building blocks, there is a trend toward using digital units to implement processing algorithms even for executing the tasks that were originally analog such as front-end communications. Microprocessors and digital signal processors rely on efficient implementation of fast arithmetic logic units to execute dedicated algorithms such as convolution and filtering [1], [2]. Adders and multipliers are most frequently and widely used arithmetic cells in realizing these processors. In most of these applications, multipliers dictate the overall performance of the system when speed and power consumption are considered as limiting factors. At the circuit design level, there is a great potential for optimization of these building blocks by voltage scaling or application of new CMOS logic styles for the implementation of its embraced combinational circuits [3]. A fast array or tree multiplier is typically composed of three subcircuits:

1) A Booth encoder for the generation of a reduced number of partial products.

2) A carry save structured accumulator for a further reduction of the partial products' matrix to only the addition of two operands.

3) A fast carry propagation adder (CPA) [4] for the computation of the final binary result from its stored

Manuscript received November 17, 2012; revised January 28, 2013.

P. Aliparast is with the Department of Electrical Engineering, Heris Branch, Islamic Azad University, Heris, Iran and the Faculty of Electrical and Computer Engineering, University of Tabriz, Tabriz, Iran (e-mail: p-aliparast@ tabrizu.ac.ir).

Z. D. Koozehkanani is with the Faculty of Electrical and Computer Engineering, University of Tabriz, Tabriz, Iran (e-mail: zdaie@tabrizu.ac.ir).

Farhad Nazari is with the Department of Electrical Engineering, Heris Branch, Islamic Azad University, Heris, Iran (e-mail: farhaad.nazari@herisiau.ac.ir). carry representation.

Among these subcircuits, the second stage of partial product accumulation, often referred to as the carry save adder (CSA) tree [5]-[7], contributes most to the overall delay and a high fraction of silicon area. Therefore, increasing the speed of CSA subcircuits is crucial to improve the performance of the multiplier. Early designs of CSA tree used the Dadda's column compression technique [8] with the 3-2 counters, or equivalently the full adders to reduce the partial product matrix. To reduce the delay of the partial product accumulation stage, 4-2 compressors have been widely employed nowadays for high speed multipliers. Because of their regular interconnection, these 4-2 compressors are ideal for the construction of regularly structured Wallace tree with low complexity [7]-[9].

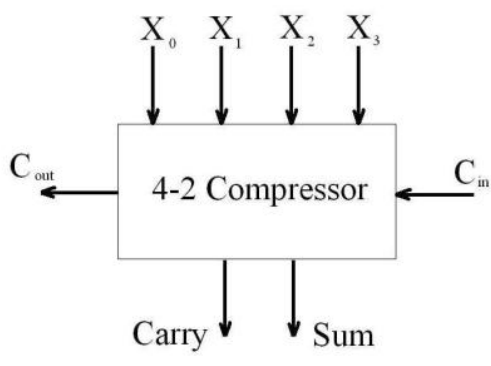

Fig. 1. Block diagram of a 4-2 compressor.

Several 4-2 compressor circuits have been proposed for high-speed applications [3]. In this paper, we begin with a brief introduction of conventional compressors which are composed of two full adders and each full adder optimized in gate level to achieve high speed. After investigating the performances of this 4-2 compressor architecture and their underlying building modules, a new very high speed current mode fully differential 4-2 compressor is proposed. The 4-2 compressors constructed with this current mode technique exhibit superior speed efficiency comparing to other configurations.

\section{The CONVEntional 4-2 COMPRESSOR STRUCTURE}

4-2 compressor has five inputs and three outputs, as shown in Fig. 1. The four inputs $X_{0}, X_{1}, X_{2}$, and $X_{3}$, and the output have the same weight. $C_{i n}$ is the output carry of preceding module and $C_{\text {out }}$, the carry output of current stage is fed to the next compressor. The output Carry is weighted one binary bit order higher. The compressor is governed by the following basic equation:

$$
X_{0}+X_{1}+X_{2}+X_{3}+C_{\text {in }}=\text { Sum }+2 .\left(\text { Carry }+C_{\text {out }}\right)
$$

Besides, to accelerate the carry save summation of the 
partial products, it is imperative that the output $C_{\text {out }}$ be independent of the input $C_{\text {in }}$.

The conventional architecture of a 4-2 compressor consists of two serially connected full adders, as shown in Fig. 2(a). Straightforward implementation of this circuit leads to a long critical path delay. Also because of uneven delay profiles of outputs from different inputs, the CSA tree constructed from such cells generates a lot of glitches. So, optimization at gate level is suggested to alleviate these problems [3]. The optimized gate level circuit for each full adder has been illustrated in Fig. 2(b). For NAND gates in this Fig, CMOS static circuits and for realizing XOR gates, transmission gate (TG) circuits have been used. In overall for implementing 4-2 compressor with this method, 72 transistors are required.
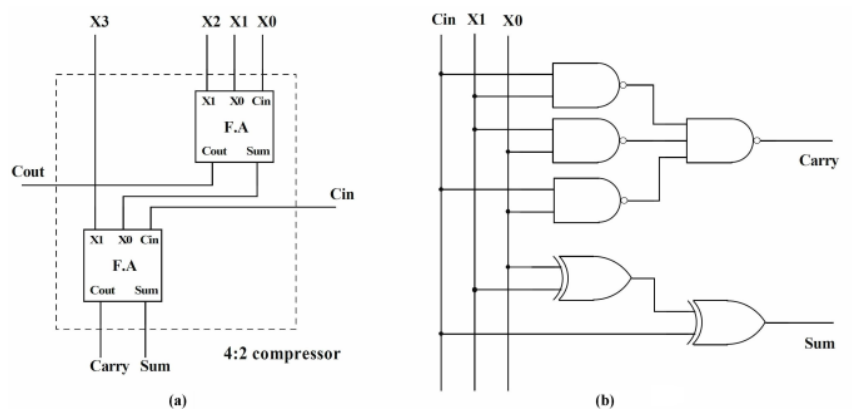

Fig. 2. (a) Convential 4-2compressor scheme, (b) gate level strucure of a full adder.

\section{Proposed New 4-2 COMPRESSOR STRUCTURE}

In this section, we describe the new method for implementing 4-2 compressor of Fig. 2(a). This method is based on current mode circuits and adds the currents in analog form.

\section{A. New Full Adder Architecture}

If we consider the operation of a full adder, we can replace it with a current mode digital to analog converter (DAC) which produces a current proportional to the inputs of full adder. Table I shows the truth table of the operation and the amount of output current. If we examine DAC current column in Table I and the state of outputs Sum and $C_{\text {out }}$ we can change it to a simpler from as shown in Table II. If we pay attention to this truth table, we can easily set the required output bits of full adder. The design procedure is as follow: according to the Table II it is enough that for currents higher than $1.5 \mathrm{I}$ with proper margin, we set $C_{\text {out }}$ to 1 and if the current is odd we will set Sum to 1 . To do this, it is enough to decrease 2I from DAC current when the DAC current is more than 2I. So in this manner, we can compare the output current of the DAC with corresponding currents and set the required bit and when DAC current is less than 2I we compare it with $0.5 \mathrm{I}$. In this case, if it is higher than $0.5 \mathrm{I}$ we will set Sum to 1 . For comparison of currents we have used two series current source that one of them works as a source and the other one works as a sink. It is clear that if the source current is more than the sink, voltage of the middle node goes to high and vice versa. Fig. 3 illustrates the proposed structure for a full adder.

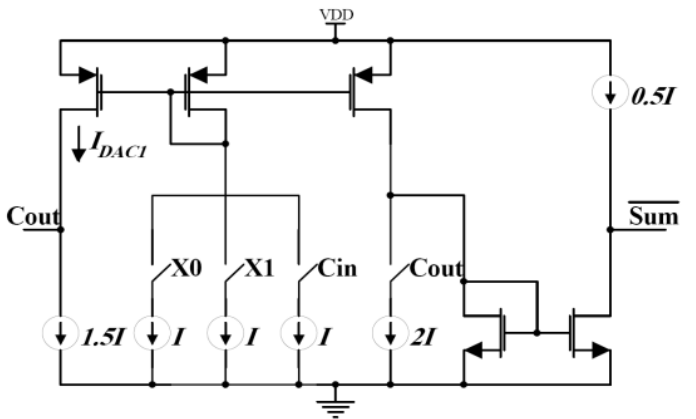

Fig. 3. Proposed new full adder structure.

\begin{tabular}{cccccc}
\multicolumn{6}{c}{ TABLE I: TRUTH TABLE OF THE FULL ADDER } \\
\hline $\boldsymbol{C}_{\boldsymbol{i n}}$ & $\boldsymbol{X}_{\boldsymbol{0}}$ & $\boldsymbol{X}_{\boldsymbol{I}}$ & $\begin{array}{c}\text { DAC } \\
\text { Current }\end{array}$ & Sum & $\boldsymbol{C}_{\text {out }}$ \\
\hline 0 & 0 & 0 & 0 & 0 & 0 \\
0 & 0 & 1 & $\mathrm{I}$ & 1 & 0 \\
0 & 1 & 0 & $\mathrm{I}$ & 1 & 0 \\
0 & 1 & 1 & $2 \mathrm{I}$ & 0 & 1 \\
1 & 0 & 0 & $\mathrm{I}$ & 1 & 0 \\
1 & 0 & 1 & $2 \mathrm{I}$ & 0 & 1 \\
1 & 1 & 0 & $2 \mathrm{I}$ & 0 & 1 \\
1 & 1 & 1 & $3 \mathrm{I}$ & 1 & 1 \\
\hline
\end{tabular}

TABLE II: SIMPLIFICATION OF TABLE I DAC Current Sum $C_{\text {out }}$

\begin{tabular}{ccc}
\hline 0 & 0 & 0 \\
I & 1 & 0 \\
2 I & 0 & 1 \\
$3 \mathrm{I}$ & 1 & 1
\end{tabular}

\section{B. New 4-2 Compressor Architecture}

Considering the structure of a 4-2 compressor which is constructed using two full adders (Fig. 2(a)). $I_{D A C 1}$ and $I_{D A C 2}$ are analog currents corresponding to the outputs of each full adder. To realize this compressor the full adder structure proposed in section II. A. has been used (Fig. 3). But note that the Sum output of first full adder is directly connected to second full adder so there is no need to current to voltage conversion. By subtracting $2 I$ from $I_{D A C 1}$ current corresponding to the Sum output of first full adder could be created and the use of an additional comparator could be avoided. Fig. 4 illustrates the proposed structure for 4-2 compressor.

At first, input bits $X_{0}, X_{1}, X_{2}$ produce the $C_{\text {out }}$ then using $C_{\text {out }}$ bit, first full adder DAC current $\left(I_{D A C 1}\right)$, also $C_{\text {in }}$ and $X_{3}$ bits, the Carry output is generated. Finally, Carry and second full adder DAC current produce the Sum bit. To understand how this circuit operates, we use one row of truth table as an example which is shown in Table III. For first full adder consider inputs as $X_{0}=1, X_{1}=0, X_{2}=1$, so in the circuit of Fig. 4, switches $X_{0}, X_{2}$ will be closed and switch $X_{1}$ will be open and it results in $I_{D A C 1}=2 I$. To generate $C_{\text {out }}$, it is enough to compare this current with $1.5 \mathrm{I}$. Because, it is higher than $1.5 \mathrm{I}$ then $C_{\text {out }}$ will be 1 . Now, for the inputs of second full adder considering $C_{i n}=1, I_{D A C 1}=2 I$ and $X_{3}=1$, causes switches 
$X_{3}$ and $C_{\text {in }}$ to be closed. Also, because of $C_{\text {out }}=1$ the switch $C_{\text {out }}$ will be closed. The current $I_{D A C 2}$ for this node will be:

$$
I_{D A C 2}=I_{D A C 1}+I+I-2 I \Rightarrow I_{D A C 2}=I_{D A C 1}=2 I
$$

Again this current is higher than 1.5I which leads to $\overline{\text { Carry }}=0$. As a results, "Carry", switch will be closed and current 2I will subtracted from $\mathrm{I}_{\mathrm{DAC} 2}$ and compared to $0.5 \mathrm{I}$. Because $0.5 \mathrm{I}$ is higher than zero will cause Sum to be 0 . For this example, inputs and the results have been summarized in Table III. The same description can be used for other sets of inputs.

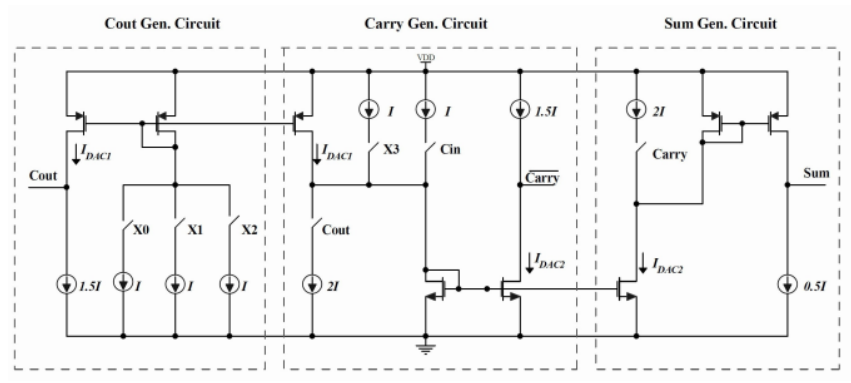

Fig. 4. Proposed new 4-2 compressor architecture.

TABLE III: TRUth TABLE For ONE Row EXAMPLE OF THE PROPOSED 4-2 COMPRESSOR

\begin{tabular}{cccccccc}
\hline$C_{\text {in }}$ & $X_{3}$ & $X_{2}$ & $X_{1}$ & $X_{0}$ & Sum & Carry & $C_{\text {out }}$ \\
\hline 1 & 1 & 1 & 0 & 1 & 0 & 1 & 1 \\
\hline
\end{tabular}

\section{Circuit Implementations of the PROPOSED 4-2 COMPRESSOR ARCHITECTURE}

As shown in Fig. 4, the proposed 4-2 compressor circuits compose of three sections ( $C_{\text {out }}$ generation circuit, Carry generation circuit and Sum generation circuit). Fig. 5 shows the $C_{\text {out }}$ generation circuit. As it is clear from the Fig. 5 each switches of $X_{0}, X_{1}$ and $X_{2}$ is replaced with a differential switch. The reason for using differential switch instead of single MOS switch is the advantages of these switches in very high speed operation and producing signal and its complement at same time. On the other hand differential switches can be switch with almost $\sqrt{2} \times \Delta V$ where $\Delta V$ is overdrive voltage of MOS transistor, so it can follow very small changes in differential input voltages. For implementation of the current sources in Fig. 4, they have been replaced with current mirror circuits. For a tradeoff between power consumption and speed, we have chosen $2.5 \mu \mathrm{A}$ for the value of I. It is clear that increase of I leads to increase of power and speed of compressor. With same method for $C_{\text {out }}$ generation circuit we can implement circuits of Carry generation and Sum generation sections. It is enough that each of switches replaces with differential switches and each current source replace with current mirror transistors. Figs. 6 and 7 show the Carry generation circuit and Sum generation circuit respectively. Fig. 8 shows the output latch scheme that has been used as output load for the proposed 4-2 compressor. Output voltage signal of this circuit can change rail to rail while its input doesn't need a rail to rail voltage signal. Thus it's useful for connected the outputs of the proposed compressor to other static CMOS logic circuits without worry about drawing static current.

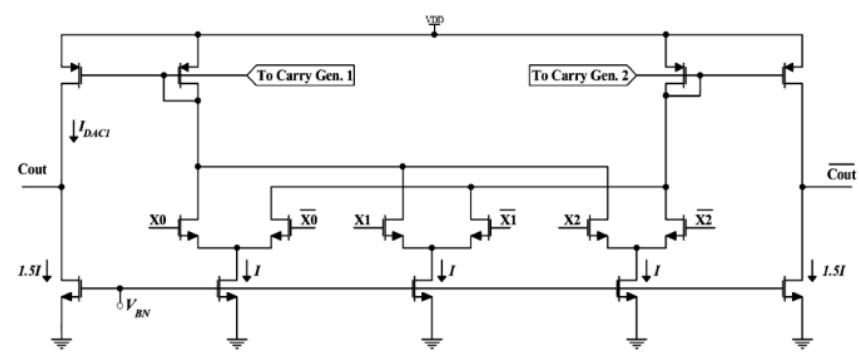

Fig. 5. Cout generation circuit of proposed 4-2 compressor.

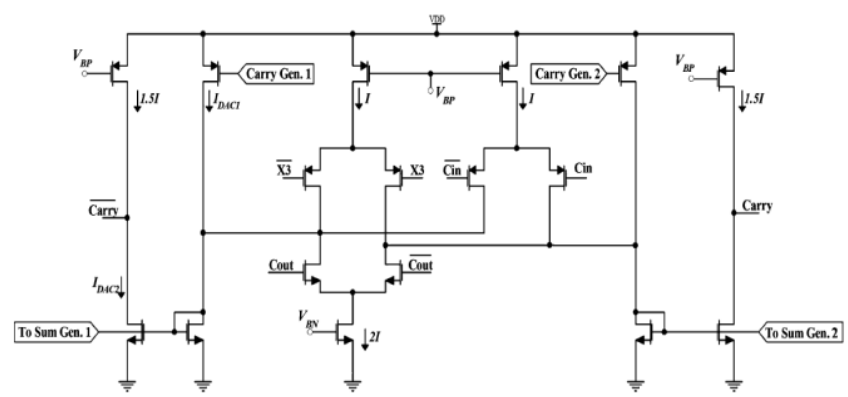

Fig. 6. Carry generation circuit of proposed 4-2 compressor.

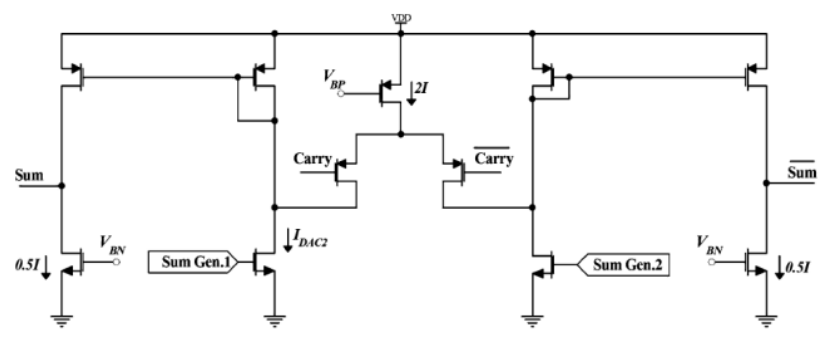

Fig. 7. Sum generation circuit of proposed 4-2 compressor.

\section{Simulation Performance}

Fig. 9 shows the simulation environments for the 4-2 compressor. Each input is driven by a minimum size inverter signal. For output load, the proposed 4-2 compressor used latch circuit that is shown in Fig. 8. Conventional compressor structure used a minimum size inverter in output as a load. This consideration provides a realistic simulation environment reflecting the compressor operation in actual applications. The simulation environments of 4-2 compressor (Fig. 9) consist of two cascaded 4-2 compressors. These compressors are running in parallel to simulate an actual compressor stage in the CSA tree. The dashed lines in Fig. 9 indicate the scenario of such potential critical paths with delay time for each of them. For delay numbers, critical path (from input bits to the Sum bit of the neighboring compressor) has been considered. The delay is measured from the earliest input signal converge with its complement to the latest output signal converge with its complement. The worst case delay is largest delay among all input data. For a fair comparison, a conventional structure using two full adders and suggested current steering structure implemented and have been 
simulated using a $65-\mathrm{nm}$ IBM CMOS process with $1.2 \mathrm{~V}$ power supply voltage. Fig. 10 shows simulation results for the proposed compressor in worse case. Random input data with a rate of $1 \mathrm{GHz}$ has been fed to the inputs of the compressor. It should be noted that the simulation frequency is not maximum operating frequency of the compressors. In fact the compressors simulated are capable of operating correctly much higher frequency than the simulation frequency.

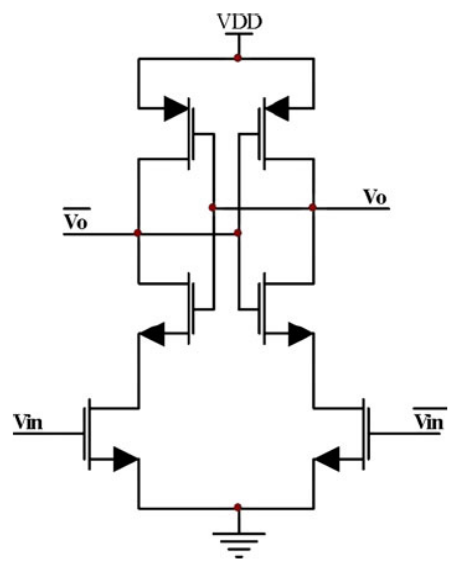

Fig. 8. The output latch circuit scheme.

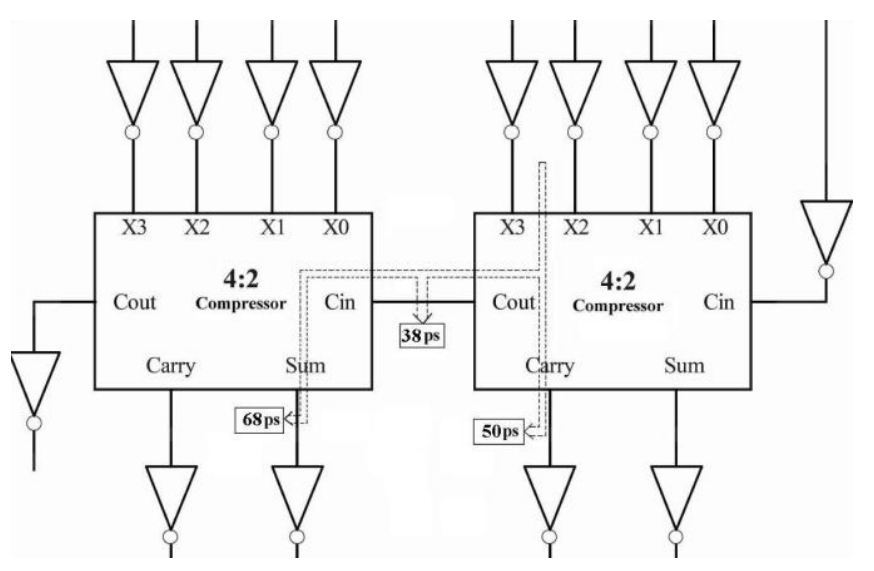

Fig. 9. 4-2 Compressor simulation environment.

Fig. 10(a) shows one of the inputs of the compressor (X0) when it changes state. In worst case condition first valid output after 38ps is Cout which is shown on Fig. 10(b). Then 50ps after input change, Carry will be valid and finally Sum output of the succeeding compressor changes its state after 68 ps. Simulation results show a reduced delay less than $68 \mathrm{ps}$ which is a considerable improvement compared to conventional architecture. Table IV summarizes the comparison of two simulated structures with explained environment in above.

TABLE IV: COMPARISON OF SIMULATED 4-2 COMPRESSORS

\begin{tabular}{c|cccc}
\hline \multirow{2}{*}{ Structure } & \multicolumn{4}{|c}{ Table Column Head } \\
\cline { 2 - 5 } & $\begin{array}{c}\text { Delay } \\
(\boldsymbol{p s})\end{array}$ & $\begin{array}{c}\text { Power } \\
(\boldsymbol{\mu W})\end{array}$ & $\begin{array}{c}\text { PDP } \\
(\boldsymbol{f J J})\end{array}$ & Number of \\
Convential & 180 & 110 & 19.8 & 72 \\
Porposed & 68 & 48 & 3.26 & 43 \\
& & & & \\
\hline
\end{tabular}
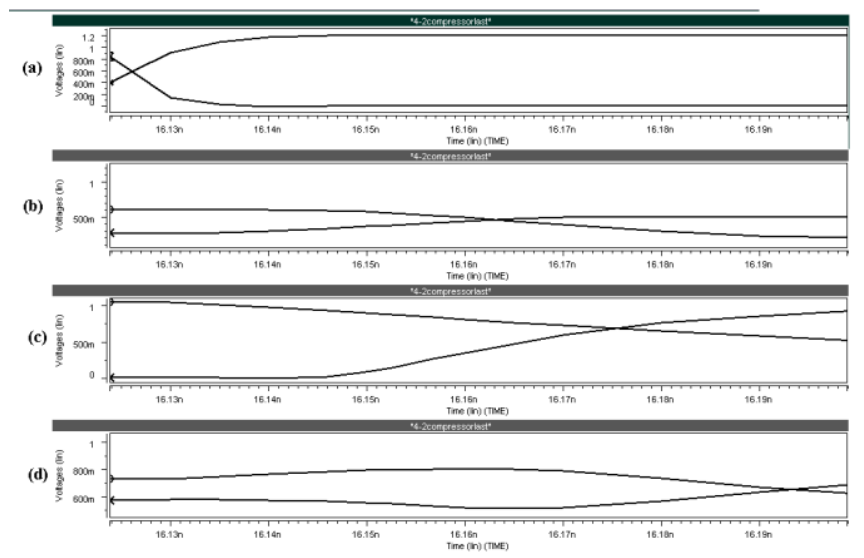

Fig. 10. Simulation results of proposed 4-2 compressor, (a) $\mathrm{X}_{0}$ and $\overline{\mathrm{X}_{0}}$, (b) $\mathrm{C}_{\text {out }}$ and $\overline{\mathrm{C}_{\text {out }}}$, (c) Carry and $\overline{\text { Carry }}$, (d) Sum and $\overline{\mathrm{Sum}}$.

\section{CONCLUSION}

In this work, a new current mode fully differential 4-2 compressor in 65-nm CMOS is presented and compared to a conventional structure compressor. Conventional structure in which the critical path delay reduction is done at gate level has higher power consumption and delay. The proposed compressor shows the highest speed performance, while maintaining lower PDP (power-delay product). Also, the proposed circuit only requires 43 transistors and most of them are minimum size hence this structure occupies smaller area than other high-speed conventional 4-2 compressors. So this is an ideal subcircuit for implementing fast digital arithmetic units.

\section{REFERENCES}

[1] K. Prasad and K. K. Parhi, "Low-power 4-2 and 5-2 compressors," in Proceedings of 35th Asilomar Conference Onsignals, Systems and Computers, vol. 1, pp. 129-133, 2001.

[2] P. J. Song and G. D. Micheli, "Circuit and architecture trade-offs for high-speed multiplication," IEEE Journal of Solid-State Circuits, vol 26, pp. 1184-1198, 1991.

[3] C. Chang, J. Gu, and M. Zhang, "Ultra low-voltage lowpower CMOS 4-2 and 5-2 compressors for fast arithmetic circuits," IEEE Journal of Transactions on Circuits and Systems Part I, vol. 51, pp. 1985-1997, 2004.

[4] C. Nagendra, M. J. Irwin, and R. M. Owens, "Area-timepower tradeoffs in parallel adders," IEEE Journal of Transactions on Circuits and Systems Part II, vol. 43, pp. 689-702, 1996.

[5] S. Hsu, S. Mathew, M. Anders, B. Zeydel, V. Oklobdzija, R. Krishnamurthy, and S. Borkar, "A 110 GOPS/W 16-bit multiplier and reconfigurable PLA loop in 90-nm CMOS," IEEE Journal of Solid-State Circuits, vol. 41, pp. 256-264, 2006.

[6] S. F. Hsiao, M. R. Jiang, and J. S. Yeh, "Design of highspeed low-power 3-2 counter and 4-2 compressor for fast multipliers," Electronics Letters, vol. 34, no. 4, pp. 341-343, 1998.

[7] D. Radhakrishnan and A. P. Preethy, "Low-power CMOS pass logic 4-2 compressor for high-speed multiplication," in Proceedings of 43rd IEEE Midwest Symposium on Circuits System, vol. 3, pp. 1296-1298, 2000.

[8] Z. Wang, G. A. Jullien, and W. C. Miller, "A new design technique for column compression multipliers," IEEE Transactions on Computers, vol. 44, pp. 962-970, 1995.

[9] S. Veeramachaneni, K. Krishna, L. Avinash, S. Puppala, and M. B. Srinivas, "Novel architectures for high-speed and low-power 3-2, 4-2 and 5-2 compressors," in Proceedings of IEEE 20th International Conference on VLSI Design, 2007. 


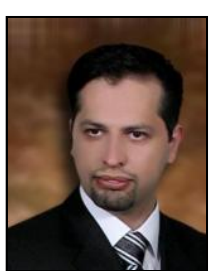

P. Aliparast was born in Tabriz, Iran. He received B.Sc. degree from Islamic AZAD University of Tabriz, Tabriz, Iran, in 2004 and M.Sc. degree from Urmia University, Urmia, Iran, in 2007, both in electronics engineering. $\mathrm{He}$ is currently $\mathrm{Ph} . \mathrm{D}$. candidate in electronics engineering in University of Tabriz, Tabriz, Iran. His research interests are analog and digital integrated circuit design for fuzzy and neural network applications, analog integrated filter design and high-speed high-resolution digital to analog converters. He is currently with Islamic AZAD University of Heris, Heris, Iran, and Integrated Circuits Research Laboratory in Tabriz University, Tabriz, Iran.

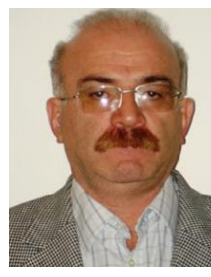

Z. D. Koozehkanany received his Ph.D. degree in Electrical Engineering from the University of Brunel University of West London, UK in 1996. He has been teaching as an assistant professor in Urmia University from 1996 to 2004 and in Tabriz University since 2004 At the time being he works as an associated professor in Electronics Department in Tabriz University and his position is Dean of ECE faculty. His current scientific interests are analog integrated circuit design including Data Converters, RF IC Design and Optical Filter Design.

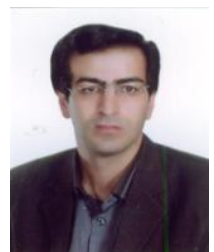

F. Nazari was born in Heris, Iran. He received B.Sc. degree from Islamic AZAD University of Tabriz Tabriz, Iran, in 2000 and M.Sc. degree from Iran University of Science and Technology, Tehran, Iran, in 2008, both in Electrical engineering. He is currently with Islamic AZAD University of Heris, Heris, Iran. 